\title{
Flora das cangas da Serra dos Carajás, Pará, Brasil: Lepidoziaceae
}

\author{
Flora of the cangas of the Serra dos Carajás, Pará, Brazil: Lepidoziaceae
}

Anna Luiza Ilkiu-Borges ${ }^{1,2}$ \& Fúvio Rubens Oliveira-da-Silva ${ }^{1}$

\begin{abstract}
Resumo
A única espécie, Telaranea nematodes, de Lepidoziaceae registrada nas áreas de canga na Serra dos Carajás, no estado do Pará, é descrita detalhadamente, ilustrada e sobre a mesma são apresentados comentários morfológicos. Palavras-chave: brioflora, hepática, FLONA Carajás, taxonomia.
\end{abstract}

\begin{abstract}
The only species, Telaranea nematodes, of Lepidoziaceae recorded for the areas of cangas in the Serra dos Carajás, Pará state, is detailed descripted, illustrated and about the species are presented morphologic comment. Key words: bryoflora, liverwort, FLONA Carajás, taxonomy.
\end{abstract}

\section{Lepidoziaceae}

Lepidoziaceae Limpr. reúne 30 gêneros, dos quais 12 ocorrem no Brasil (Gradstein et al. 2001; Gradstein \& Costa 2003; Söderström et al. 2016). No país, foram registradas 48 espécies (Gradstein \& Costa 2003). Reconhecidamente heterogênea, essa família reúne plantas folhosas, raramente talosas, com filídios íncubo, súcubo ou com inserção transversal, alternados, usualmente dividido em diversos segmentos ou dentes, raramente não dividido e anfigastros desenvolvidos (raro reduzidos) (Gradstein \& Costa 2003; Gradstein \& Ilkiu-Borges 2009). Na Serra dos Carajás foi registrada apenas uma espécie do gênero Telaranea (Spruce) Schiffn.

\section{Telaranea (Spruce) Schiffn.}

Esse gênero compreende 33 espécies aceitas (Söderström et al. 2016), das quais apenas três foram registradas no Brasil (Costa \& Peralta 2015). Telaranea é representado por plantas com filídios com inserção transversal ou súcuba, divididos em 2-4 filamentos unisseriados ou consistindo de apenas um filamento (Gradstein \& Ilkiu-Borges 2009).
1.1. Telaranea nematodes (Gottsche ex Austin) M.A.Howe, Bull. Torrey Bot. Club 29 (5): 284, 1902. Cephalozia nematodes Gottsche ex Austin, Bull. Torrey Bot. Club 6 (52): 302, $1879 . \quad$ Figs 1a-c

Plantas 0,6-1 $\mathrm{mm}$ de largura, verde-pálido a verde-esbranquiçado. Filídios filiformes, consistindo de 2-3(-4) filamentos uniseriados, (3-)4-6 células de comprimento, conectadas na base. Anfigastros consistindo de 2(-3) filamentos curtos de 1-3 células de comprimento.

Material selecionado: Canaã dos Carajás, S11C, 6²2'58,2”S, 50²3'08,3'W, 29.IV.2015, A.L. IlkiuBorges et al. 3482 (MG). Parauapebas, N5, $6^{\circ} 06^{\prime} 18,1$ 's, 5007'49,3”W, 27.IV.2015, A.L. Ilkiu-Borges et al. 3378 (MG).

Essa espécie é reconhecida pelos filídios filamentosos, profundamente subdivididos desde a base. É geralmente coletada em locais úmidos e sombreados, desde florestas de planície a florestas de altitude sobre solo, liteira, húmus, rochas, troncos vivos e em decomposição (Gradstein \& Costa 2003; Gradstein \& Ilkiu-Borges 2009). Na Serra dos Carajás, essa espécie ocorreu sobre tronco vivo ou em decomposição e sobre rocha de ferro, em locais

\footnotetext{
${ }^{1}$ Museu Paraense Emílio Goeldi, Av. Magalhães Barata 376, São Braz, 66040-170, Belém, PA, Brasil.
} 

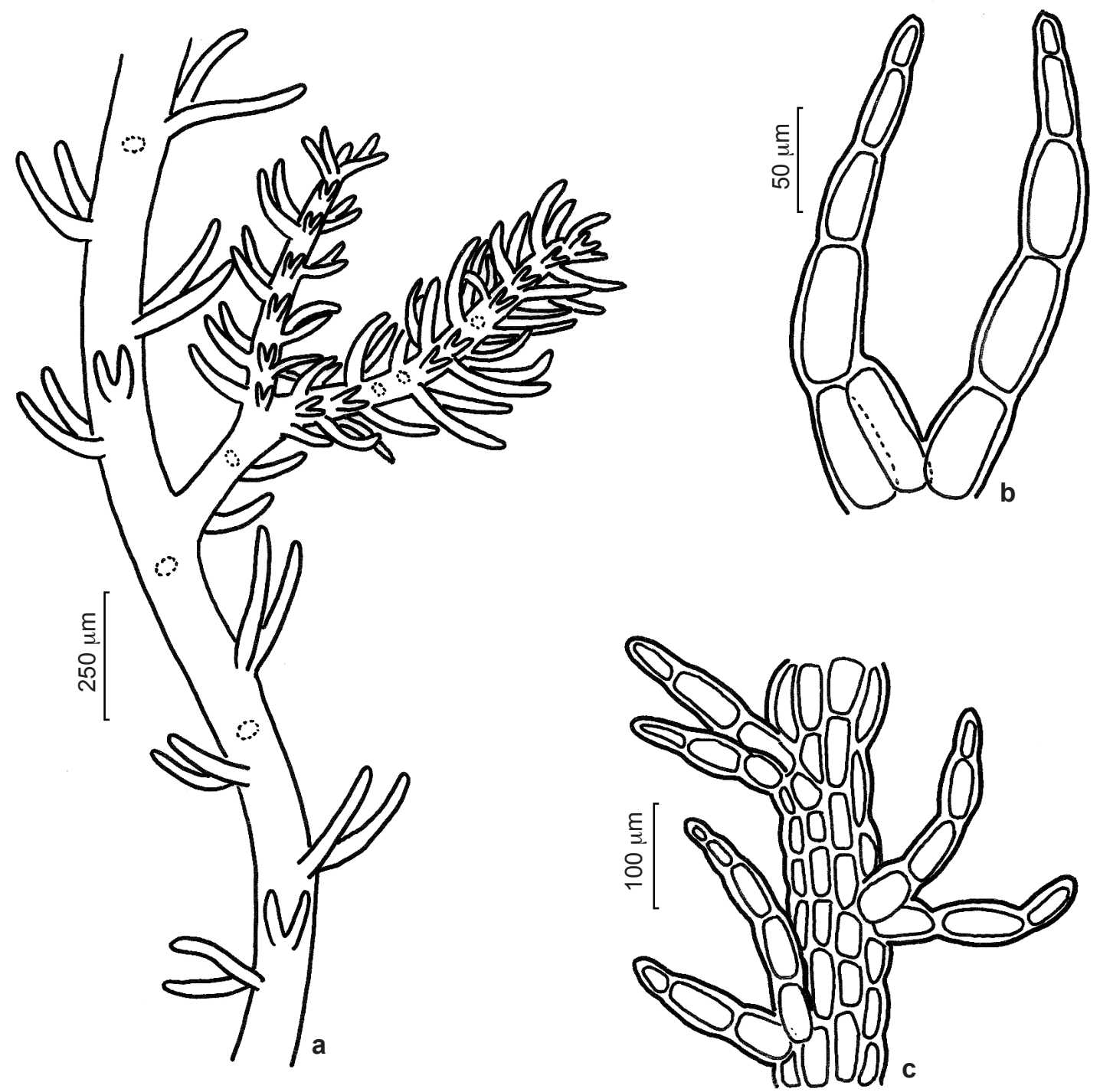

Figura 1 - a-c. Telaranea nematodes - a. hábito; b. filídio; c. seção do caulídio.

Figure 1 - a-c. Telaranea nematodes - a. habit; b. leaf; c. sector of a stem.

sombreados, seja em florestas sobre canga ou às margens de cursos d'água na canga com poucas árvores.

Distribuída na América Tropical, África e Europa Ocidental. No Brasil ocorre nos estados do AC, AM, BA, CE, DF, ES, GO, MG, MS, MT, RJ, RO, RS, SC, SE, SP. Serra dos Carajás: Serra Sul: S11-C e Serra Norte: N5.

\section{Agradecimentos}

Agradecemos ao Museu Paraense Emílio Goeldi e Instituto Tecnológico Vale, a infraestrutura e demais apoios fundamentais para o desenvolvimento deste trabalho; assim como à Dra. Ana Maria Giulietti Harley e ao Dr. Pedro Viana, coordenadores do projeto conveniado MPEG/ITV/ FADESP (01205.000250/2014-10) e ao projeto aprovado pelo CNPq (processo 455505/2014-4), o financiamento; ao ICMBio, em especial ao biólogo Frederico Drumond Martins, a licença de coleta concedida e suporte nos trabalhos de campo; ao CNPq, a bolsa de Iniciação Científica concedida ao segundo autor e a bolsa de Produtividade em Pesquisa concedida à primeira autora. 


\section{Referências}

Costa, D.P. \& Peralta, D.F. 2015. Bryophytes diversity in Brazil. Rodriguésia 66: 1063-1071.

Gradstein, S.R. \& Costa, D.P. 2003. The Hepaticae and Anthocerotae of Brazil. Memoirs of The New York Botanical Garden 87: 1-318.

Gradstein, S.R.; Churchill, S.P. \& Salazar-Allen, N. 2001. Guide to the Bryophytes of tropical America. Memoirs of The New York Botanical Garden 86: 1-577.

Gradstein, S.R. \& Ilkiu-Borges, A.L. 2009. Guide to the plants of Central French Guiana. Part 4. Liverworts and Hornworts. Memoirs of The New York Botanical Garden 76: 1-140.
Söderström, L.; Hagborg, A.; von Konrat, M.; Bartholomew-Began, S.; Bell, D.; Briscoe, L.; Brown, E.; Cargill, D.C.; Costa, D.P.; CrandallStotler, B.J.; Cooper, E.D.; Dauphin, G.; Engel, J.J.; Feldberg, K.; Glenny, D.; Gradstein, S.R.; He, X.-L.; Heinrichs, J.; Hentschel, J.; IlkiuBorges, A.L.; Katagiri, T.; Konstantinova, N.A.; Larraín, J.; Long, D.G.; Nebel, M.; Pócs, M.; Puche, F.; Reiner-Drehwald, E.; Renner, M.A.M.; Sass-Gyarmati, A.; Schäfer-Verwimp, A.; Moragues, J.G.S.; Stotler, R.E.; Sukkharak, P.; Thiers, B.M.; Uribe, J.; Váňa, J.; Villarreal, J.C.; Wigginton, M.; Zhang, L. \& Zhu, R.-L. 2016. World Checklist of hornwrts and liverworts. Phytokeys 59: 1-828. 
